\title{
Uma Abordagem do Scrum para Gestão de Tempo, Produtividade e Bem-Estar
}

\author{
Carlos Fran Ferreira Dantas \\ Instituto Federal \\ do Rio Grande do Norte (IFRN) \\ Apodi-RN, Brasil \\ carlos.dantas@ifrn.edu.br
}

\author{
Rosane Targino de Medeiros \\ Universidade Federal \\ do Rio Grande do Norte (UFRN) \\ Natal-RN, Brasil \\ rosanetarginomd@gmail.com
}

\author{
Fernanda Ligia Leonardo \\ Marques \\ Instituto Federal \\ do Rio Grande do Norte (IFRN) \\ Apodi-RN, Brasil \\ fernanda.marques@ifrn.edu.br
}

\begin{abstract}
Scrum has been a framework widely used in the management of software projects in the perspective of agile development. The success and simplicity of Scrum has motivated its adoption in other fields, such as its version for education - eduScrum. In this sense, this work presents a Scrum approach to time and productivity management, incorporating positive psychology practices and techniques in order to improve the wellness of users. In an initial experiment, proposed as a challenge to volunteers, an impact on the positive emotions and subjective well-being of about $50 \%$ of the participants was obtained. Participants' reports indicate that the approach contributed to better planning, greater organization and focus on activities, in addition to promoting the well-being of participants with exercises related to optimism and gratitude.
\end{abstract}

\section{KEYWORDS}

Scrum, Desenvolvimento Ágil, Gestão de Tempo, Bem-estar Subjetivo, Psicologia Positiva

\section{INTRODUÇÃO}

No contexto dos estudantes concluintes, principalmente do ensino médio, observa-se maior cobrança por resultados e aumento das atividades, pois, ao mesmo tempo que realizam as atividades comuns do ano letivo em curso, preparam-se para os exames de ingresso à universidade. Além disso, tais jovens passam por um período de decisão quanto à escolha de carreira e definição de planos, como a possibilidade de inserção no mundo do trabalho.

Atrelado ao contexto destes estudantes, está o cenário pandêmico durante o ano letivo de 2020, que inclui as incertezas pertinentes à modalidade de ensino remoto adotada em caráter emergencial pelas instituições de ensino. As dúvidas referentes ao contexto de ensino, somado ao confinamento social, intensificaram a incidência de sofrimento psíquico nos mais diversos graus, vindo a desencadear sentimentos desfavoráveis e emoções em descompasso, os quais afetam diretamente a execução das tarefas pelos estudantes, culminando na redução da produtividade e bem-estar dos discentes [Moreira et al. 2020; Maia and Dias 2020].

Tais mudanças têm causado sentimentos de autocobranças, distorções cognitivas, infelicidade, ansiedade em diferentes níveis que, em situações extremas, desencadeiam sintomas de transtorno depressivo em diferentes intensidades [Mattos 2020].

Diante desse panorama, foi questionado aos discentes de uma turma concluinte do curso técnico integrado em Informática sobre quais as principais dificuldades e desafios do momento para eles.
Como resultado, foi elaborada uma nuvem de termos presentes nas respostas (Figura 1).

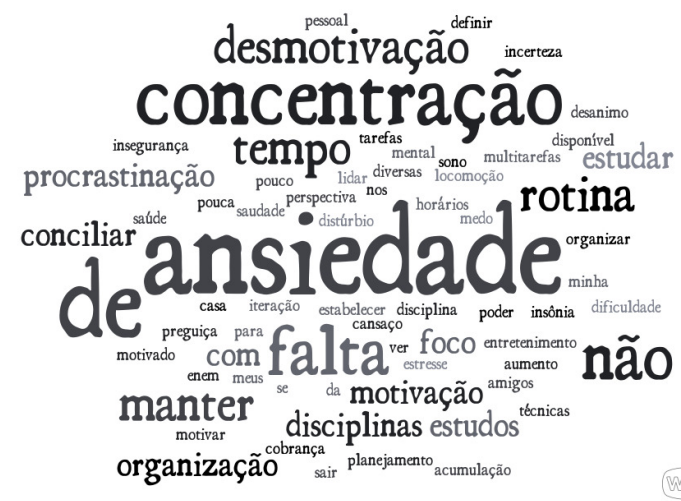

Figura 1: Diagnóstico realizado com estudantes concluintes de curso técnico integrado em Informática

Percebeu-se que os discentes apresentam ansiedade (em algum nível), além de relatarem problemas relativos à gestão de tempo e produtividade, como dificuldade em estabelecer uma rotina, desmotivação, ausência de foco, procrastinação, desânimo, insônia e dificuldades de concentração e organização.

Diante do cenário, foi proposto aos discentes na disciplina Projeto de Desenvolvimento de Software, que aborda conceitos e práticas de Engenharia de Software, uma abordagem do Scrum para gestão de tempo e produtividade, inserindo aspectos da psicologia positiva.

Por psicologia positiva [Seligman and Csikszentmihalyi 2000], define-se a ciência que se dedica a estudar os traços e experiências positivos dos indivíduos, de modo a objetivar o foco nos aspetos positivos e promover qualidade de vida das pessoas, além de atuar de forma preventiva em relação às psicopatologias; desse modo, proporciona significados salutares aos objetivos e traços positivos das pessoas.

A proposta de uma abordagem do Scrum para gestão de tempo e produtividade tem como objetivo munir os participantes de ferramentas para gerir o tempo e a produtividade, agregando as práticas e constructos da psicologia positiva - manejo e gerenciamento das emoções e sentimentos positivos, gratidão, otimismo, altruísmo, autocompaixão -, visto a potencialização dos resultados no planejamento, organização e execução Scrum.

As seções seguintes deste trabalho expõem conceitos da psicologia positiva e de bem-estar subjetivo (Seção 2), apresenta o 
framework Scrum em mais detalhes (Seção 3), relata trabalhos semelhantes (Seção 4) e detalha a abordagem proposta (Seção 5), incluindo resultados e considerações de um estudo inicial de utilização da abordagem apresentada neste documento.

\section{A PSICOLOGIA POSITIVA E O BEM-ESTAR SUBJETIVO}

A Psicologia Positiva é um campo da psicologia que investiga os sentimentos, as emoções, as instituições e os comportamentos positivos; e, tem como propósito a felicidade humana, também referenciado como bem-estar subjetivo [Seligman and Csikszentmihalyi 2000; Camalionte and Boccalandro 2017].

[Seligman and Csikszentmihalyi 2000] expõem que é possível experimentar da felicidade por meio de três elementos: emoções positivas, o engajamento (flow) e a vida significativa.

As emoções positivas tratam-se das sensações como gratidão, otimismo, diversão, orgulho, dentre outras.

O estado de flow, engajamento, remete ao estado em que a pessoa está envolvida em uma atividade em alto nível de concentração, absorvida pela momento e atividade.

A vida significativa tem forte relação com o altruísmo, o qual se relaciona com o servir e compreender sua função em algo maior, estabelecendo como propósito de vida.

O bem-estar subjetivo pode ser determinado com base no nível de satisfação com a vida, a frequência das emoções positivas e a frequência das emoções negativas. Quanto maior o nível de satisfação com a vida (em geral, traduzido pelas pessoas como bem-estar ou felicidade) e a frequência das emoções positivas, maior será o nível de bem-estar subjetivo de uma pessoa [Zanon et al. 2020].

Segundo [Sheldon and Lyubomirsky 2019], é possível aumentar os níveis de felicidade por meio de comportamentos intencionais que envolvam gratidão, empatia e altruísmo.

Nesses termos, vislumbra-se as ações que remetam à promoção de emoções positivas como gratidão e otimismo, ao passo que contribuam para a busca de sentido e engajamento em atividades ligadas aos objetivos pessoais, que podem contribuir para o bem-estar e a saúde mental das pessoas.

\section{O FRAMEWORK SCRUM}

O Scrum é um framework estruturado para apoiar o desenvolvimento de produtos e serviços em todos os tipos de indústrias e em qualquer tipo de projeto, independentemente de sua complexidade [SCRUMStudy 2016].

O Scrum é fundamentando em seis princípios que caracterizam o funcionamento do framework. São os princípios: (1) Desenvolvimento Iterativo, (2) Controle de Processo Impírico, (3) Autoorganização, (4) Colaboração, (5) Priorização orientada a valor e (6) Time-boxing.

O Scrum permite englobar vários processos ou técnicas, de forma a adaptá-lo ao projeto para obter maior eficiência [Schwaber and Sutherlandn 2017].

O fluxo do Scrum para uma sprint é exposto na Figura 2.

O Scrum possui apenas dois artefatos e cinco eventos, os quais podem ser definidos como:

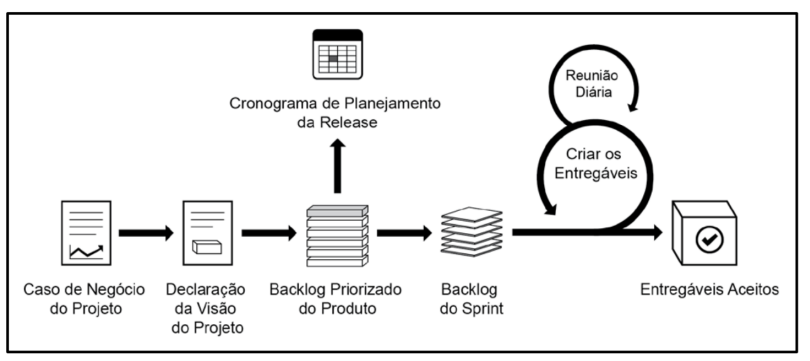

Figura 2: Fluxo do Scrum para uma sprint (Fonte: [SCRUMStudy 2016])

- Sprint: um time-box, intervalo de tempo preestabelecido para um ciclo de execução de tarefas com o objetivo de realizar uma etapa do projeto que, ao final, terá uma entrega de valor para o cliente/usuário. Uma sprint, em geral, tem duração de uma a quatro semanas.

- Planejamento da Sprint: momento no qual são definidos os itens alvos da execução da sprint.

- Reunião Diária: é uma reunião, estilo stand up, na qual cada membro da equipe comunica em que está trabalhando atualmente e quais as dificuldades existentes, de maneira a possibilitar colaboração dos demais membros.

- Revisão da Sprint: caracteriza-se por um momemnto de apresentação dos resultados da sprint ao cliente/usuário. Nesse momento, são coletados alguns feedbacks e atualizado o backlog do produto.

- Retrospectiva da Sprint: define-se por uma reunião de equipe do projeto, com intuito de avaliar a execução da sprint e identificar pontos positivos e pontos negativos, além de definir ações para aperfeiçoar o processo de desenvolvimento.

Os artefatos do Scrum são o Backlog do Produto e o Backlog da Sprint. O termo backlog refere-se ao cojunto de itens a serem tratados no projeto. Assim, o Backlog do Produto remete ao conjunto de todos os itens do projeto, os quais são organizados em uma lista priorizada, e o Backlog da Sprint refere-se ao conjunto de itens que serão tratados no próximo ciclo de execução do projeto.

User story são descrições simples e concisas, escritas de modo que o cliente expresse suas necessidades e a equipe do projeto utilize-as para definição do backlog do produto [Brod 2015].

O Scrum estabelece o conceito de "Definição de Pronto", uma convenção estabelecida pelos envolvidos no projeto para conclusão de um item do backlog ou objetivo da sprint corrente [SCRUMStudy 2016; Schwaber and Sutherlandn 2017; Brod 2015].

O Scrum é um framework para gestão de projetos com notório reconhecimento de sua qualidade e dentre os vários motivos de sua grande utilização, estão a adaptabilidade, a melhoria contínua e o ritmo sustentável proporcionado aos projetos geridos.

\section{TRABALHOS RELACIONADOS}

Em [Starr and Starr 2008], David Starr e Eleanor Starr relatam a utilização do Scrum por três anos na família. 
$\mathrm{Na}$ abordagem, backlog é representado por uma lista de itens que devem ser cumpridos pela manhã por cada membro da família; a sprint tem duração de uma semana; a reunião diária antes da saída para escola/trabalho; e, a revisão e retrospectiva são realizadas em uma reunião semanal da família.

Segundo [Starr and Starr 2008], a utilização do Scrum aumentou a comunicação na família melhorando comportamentos das crianças e criando um sentimento positivo de equipe. As crianças foram encorajadas a participarem do processo e passaram a contribuir com ideias para melhorar a dinâmica na família. Por fim, os autores ressaltam a necessidade de se manter aberto a mudanças (adaptabilidade) e que o objetivo de mudanças na família é a felicidade, uma família saudável.

As experiências de David Starr e Eleanor Starr inspiraram [Feiler 2013] que em seu livro expõe as suas experiências com o Scrum e os princípios do Manifesto Ágil [Beck et al. 2001] em sua família.

Para [Feiler 2013], equipes autogeridas, revisões frequentes e feedbacks constantes são alguns dos conceitos do desenvolvimento ágil vislumbrados com a adoção do Scrum na rotina da família.

[Feiler 2013] reforça que as práticas incorporadas na rotina da família aumentaram a comunicação, ajudaram a aproximar os membros e a reduzir o estresse.

[Sutherland 2016] exemplifica o planejamento de um projeto no Scrum utilizando-se da realização de um casamento - expondo a utilização do framework em um contexto diferente do desenvolvimento de software.

Em [Souza 2018], uma série de registros expõem a utilização do Scrum para gerir um projeto pessoal - projeto de leitura. [Souza 2018] relata como se utilizou dos eventos do Scrum no seu projeto de leitura, aborda os momentos do fluxo de uma sprint e discute aspectos dos princípios do Scrum.

[Sutherland 2016] dedica um captítulo inteiro de seu livro para tratar sobre felicidade (capítulo 7), abordando desde motivos para se preocupar com o bem-estar das pessoas envolvidas em um projeto à técnica que propõe para medir a felicidade dos membros da equipe. Tal técnica, alinhada ao kaizen (melhoria contínua), propõe algumas poucas perguntas para que cada membro responda e relacione-as ao bem-estar na empresa, como se sente no que concerne ao seu papel em relação à empresa, e o que poderia ser feito para deixá-lo mais feliz.

Os questionamentos propostos por [Sutherland 2016] remetem aos conceitos da psicologia positiva e permitem aos membros uma reflexão sobre o propósito na empresa/trabalho e seu bem-estar subjetivo no ambiente cuja permanência, em média, é de um terço do dia.

O Scrum também tem uma abordagem dedicada à aprendizagem.

O eduScrum apresenta-se como um modelo que combina o Scrum ao processo de ensino-aprendizagem (projetos colaborativos de aprendizagem) [Delhij et al. 2016].

Em paralelo ao Scrum, o eduScrum insere dois novos eventos: Formação de equipe e Reflexão pessoal.

O cuidado na formação das equipes visa o equilibrio de habilidades/competências, genêros e o rodízio da composição das equipes.

Quanto a reflexão pessoal, o evento faz parte da retrospectiva da sprint, onde os discentes avaliam a metodologia e métodos de trabalho da equipe e, em seguida, cada membro avalia os demais e a si mesmo acerca das habilidades e pontos de melhoria.
O eduScrum amplia a "definição de pronto" do Scrum, com a "Definição de Diversão", considerando que o trabalho deve ser agradável para os envolvidos e que tal fator é impactante para o melhor aproveitamento da aprendizagem.

Diante das adaptações expostas, é perceptível o potencial do Scrum em diferentes contextos e projetos. As propostas apresentadas, anteriormente, exploram alguns aspectos de bem-estar e emoções positivas como, por exemplo, a felicidade citada por [Sutherland 2016] e a definição de diversão do eduScrum.

No campo da gestão de tempo e produtividade, o método globalmente conhecido é o GTD (GGetting Things Done) de David Allen [Allen 2005].

O GTD tem por objetivo ordenar atividades e tarefas do cotidiano e proporcionar maior produtividade através da organização de um fluxo de trabalho e eliminação de distrações. No entanto, o método não trata de aspectos que se relacionam ao bem-estar subjetivo ou às emoções positivas, referindo-se a tais aspectos apenas na motivação de seu uso, colocando-se como o método que proporciona ordem ao caos na vida pessoal e profissional.

\section{GESTÃO DE TEMPO E PRODUTIVIDADE COM SCRUM}

Como visto na exposição dos trabalhos relacionados, o Scrum pode ser facilmente utilizado na gestão de projetos de outros campos, incluindo projetos pessoais. Desta forma, pode-se estabelecer as seguintes instruções para uma abordagem do Scrum na gestão de tempo e produtividade pessoal:

- Backlog do Produto - todas as tarefas e compromissos relativos às atividades da pessoa.

- Backlog da Sprint - são aqueles itens (tarefas e compromissos) priorizados para a semana (sprint com duração de uma semana).

- Planejamento da Sprint - momento de planejamento da semana incluindo a priorização das tarefas e organização dentro de uma jornada de atividades. No campo da gestão de tempo e produtividade, em geral, recomenda-se o planejamento de 6 horas de atividades diárias.

- Reunião Diária - momento, no início das atividades diárias, para visualização do que precisa ser feito, replanejamento, caso necessário, definir nova priorização e/ou organização do dia (horários, trajetos, outros).

- Revisão da Sprint - tempo dedicado à avaliação da semana quanto ao cumprimento das tarefas e compromissos, além de atualização do backlog. Este momento é seguido da retrospectiva da sprint.

- Retrospectiva da Sprint - um intervalo de tempo para reflexão sobre as técnicas, estratégias e metodologias utilizadas. É um momento de aperfeiçoamento e autoavaliação, assim como, também, pode servir de momento para busca ou reafirmação de sentido/propósito e compromissos pessoais com objetivos e sonhos.

Em relação ao Caso de Negócio do Projeto, Declação da Visão do Projeto e Cronograma de Planejamento da Realease, pode-se indicar que são representados pela definição clara das atividades (estudante, profissional, hobbies etc) que requerem o cumprimento de tarefas e comprommissos associados; objetivos e metas. 
Nesta proposta de gestão de tempo e produtividade com Scrum, recomenda-se a listagem de objetivos e criação de metas com estimativas de realização. Nesse sentido, instrui-se a criação de lista de objetivos anuais (para os próximos 3, 5, 10 anos) e a elaboração de metas distribuídas nos 12 meses para o ano corrente, apresentandose como um planejamento de releases.

O planejamento de release é também um instrumento de motivação, servindo como lembrete e passível de mudanças - seja acrescentando novos objetivos/metas ou removendo de acordo com as descobertas individuais através da autoavaliação e reflexões proporcionadas pelas retrospectivas e acontecimentos da vida.

A abordagem do Scrum na gestão de tempo e produtividade permite a inserção de várias técnicas no planejamento e execução das sprints, como a necessidade de definição de prazos para as tarefas, lidar com o perfeccionismo desnecessário, estabelecendo expectativas ou uma definição de pronto, lidar com a procrastinação aplicando técnicas como Efeito Doberman ou técnica dos 3 segundos.

$\mathrm{Na}$ abordagem proposta, o bem-estar está atrelado desde ao cuidado em incluir no planejamento as atividades de lazer e descanso, equilibrando o tempo dedicado as atividades e o cuidado pessoal; até a retrospectiva, na qual se deve avaliar a semana quanto aos métodos utilizados e as emoções, buscando exercitar a gratidão e o otimismo que auxiliam na regulação do bem-estar subjetivo.

\subsection{Experimento inicial}

Com o objetivo de realizar uma avaliação inicial da abordagem do Scrum na gestão de tempo e produtividade, os conceitos e técnicas foram organizados como um desafio proposto aos discentes concluintes do curso técnico integrado em informática.

Tal desafio foi proposto como uma atividade livre (sem avaliação), sendo solicitado que realizassem o preenchimento de um formulário de autoavaliação referente aos aspectos de organização e emoções positivas.

O formulário deveria ser preenchido antes de iniciar e ao finalizar o desafio, no intuito de identificar alterações no estado emocional dos participantes. Além das respostas ao formulário, foi solicitado na etapa final da atividade, um feedback (em áudio) sobre o desafio.

Devido ao caráter de aplicação da abordagem e público-alvo da proposta, os termos do Scrum não foram citados de forma explícita, focando no entendimento e adoção do fluxo, sem demais exigências com a nomenclatura técnica.

Tendo em vista a simplificidade de produção e o acesso ao conteúdo, o formato podcast foi adotado para as instruções do desafio. Utilizou-se da plataforma Anchor ${ }^{1}$ que realiza a distribuição em várias outras plataformas de streaming de áudio.

O desafio foi estruturado em 7 episódios rápidos (duração de 3 a 10 minutos) com uma tarefa a ser cumprida dia a dia durante uma semana, proporcionando uma mudança gradativa e oportunidades de reflexão em cada um dos tópicos abordados.

Os episódios produzidos foram:

(1) Sobre o Desafio Rainbow

Apresentação do desafio, objetivos e a preparação para os 7 dias de desafios. Este episódio propõe a reflexão e clareza quanto aos objetivos e atividades da pessoa. Neste momento,

\footnotetext{
${ }^{1}$ https://anchor.fm
}

o desafiado deve descriminar uma lista de seus objetivos e metas, algo semelhante à uma declaração de visão do projeto no Scrum. Além disso, recomenda-se a listagem de todas as tarefas à serem realizadas, na perspectiva de se construir o backlog e um planejamento de releases (organização de metas e objetivos em blocos de tempo).

(2) Planejar Semana

Sobre como planejar a semana, dia a dia, organizando as tarefas da semana. Prazos e priorização das tarefas. Neste episódio, os participantes são instruídos à priorizar tarefas por valor (importância e urgência), identificar turno que considera ser mais produtivo e a incluir atividades de lazer e exercícios físicos no planejamento, contribuindo para a saúde e bem-estar. O planejamento da semana tem por objetivo melhorar a organização e ajudar no foco, alinhando a execução das tarefas com os objetivos e metas definidos. Caracteriza-se como o planejamento da sprint.

(3) Planejar Distrações

Alerta sobre distrações e como identificar e eliminar as fontes dessas distrações. Apresenta a técnica pomodoro e orienta sobre identificar e planejar intervalos para as distrações. O objetivo deste desafio é fazer uma mudança na postura, proporcionando maior foco no que precisa ser realizado e planejar intervalos que podem ser gratificantes. Com base na técnica pomodoro, o desafio implica na estimativa de tempo/esforço. No tocante ao bem-estar, propõe-se melhor utilização dos intervalos com pequenos hobbies e atividades que contribuem para o bem-estar, como brincadeiras com animais de estimação e abraços em pesssoas queridas próximas (mãe, pai, filhos, parceiro, etc).

\section{(4) Planejar Trajetos}

Proposta de organizar dias e horários para realizar deslocamentos e exibir dicas de como aproveitar, de forma produtiva e prazerosa, o tempo em salas de espera e translados.

\section{(5) Planejar Preocupações}

Apresentar motivações para o controle da ansiedade, com o planejamento de preocupações, de forma a equilibrar o planejar do futuro e viver o presente.

\section{(6) Seja Otimista}

Instrui para a busca do otimismo colocando a avaliação como uma oportunidade de melhoria, sentido e perspectivas mais adequadas. Relaciona-se com a motivação necessária para cumprimento das tarefas na sprint e sentido de execução do projeto.

\section{(7) Seja Grato}

Expõe a gratidão como contribuidor para o sucesso e apresenta técnicas/exercícios como Diário da Gratidão, para auxiliar no vislumbre de motivos para ser grato. Assim como o desafio anterior, este desafio relaciona-se com a motivação e cumprimento da sprint e execução do projeto.

(8) Avalie!

É enfatizada a importância da avaliação frequente e pertinente ao que está sendo trabalhado e, o convite a avaliar a experiência do desafio. Neste episódio final, avalia-se os potnos positivos e negativos da semnaa, atualiza-se as tarefas à serem realizadas e definido que medidas devem ser tomadas 
para aperfeiçoar todo o processo (proposta de restrospectiva do Scrum).

O primeiro episódio foca na organização das ideias criando uma visão por meio de objetivos e metas. Como estimulo, este exercício permite aos participantes reconhecerem que podem alcançar seus objetivos e, para tal, pequenos esforços devem ser despendidos nas tarefas para alcance das metas que poderão levá-lo a atingir o objetivo pretendido.

Os episódios 2, 3, 4 e 5 relacionam-se com o conceito de Sprint e planejamento da sprint.

Nos episódios 6, 7 e 8 são tratadas questões mais relacionadas a retrospectivas, com um espaço destinado às emoções positivas otimismo e gratidão.

Estudos na área da psicologia positiva reforçam que sentimentos como gratidão e otimismo contribuem para nosso bem-estar, comumente referidos como felicidade.

Segundo pesquisas relatadas em [Achor 2017], as pessoas felizes são pessoas mais produtivas e que alcançam o que definem como sucesso (pessoas bem-sucessedidas).

Nessa perspectiva, acredita-se que a instrução das pessoas para a gestão de tempo e a manutenção do bem-estar contribuem não só para a produtividade, mas também, para a saúde mental das pessoas.

\subsection{Resultados alcançados}

O desafio foi realizado por oito discentes, cujas respostas ao questionário sugerem impacto quanto ao otimismo, gratidão e bem-estar subjetivo dos participantes.

As autoavaliações dos participantes são indicadas nos gráficos figuras 3 , 4 e 5 .

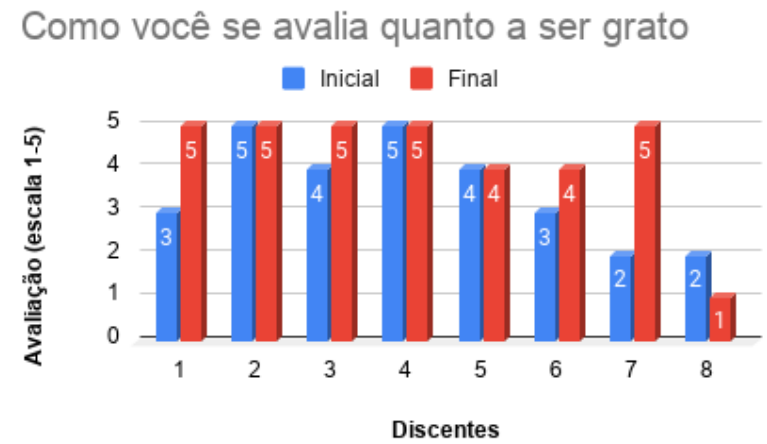

Figura 3: Autoavaliações dos participantes quanto à gratidão

Os resultados expõem que $50 \%, 75 \%$ e $50 \%$ dos participantes tiveram impacto positivo quanto à gratidão, otimismo e bem-estar. Foi observado que em apenas um dos participantes houve alterações negativas nas autoavaliações.

O participante cuja alteração foi negativa, teve a situação investigada e identificou-se algumas problemáticas ligadas à saúde da família do discente, exigindo que o participante assumisse responsabilidades e se afastasse de suas atividades acadêmicas e pessoais.

\section{Como você se avalia quanto a ser otimista}

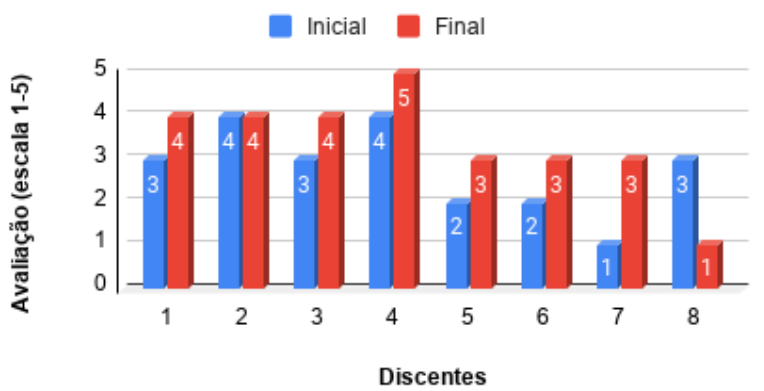

Figura 4: Autoavaliações dos participantes quanto à otimismo

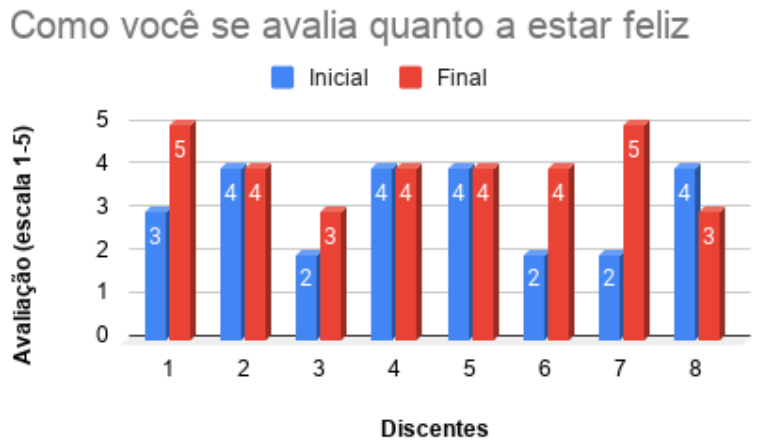

Figura 5: Autoavaliações dos participantes quanto à felicidade (bem-estar subjetivo)

O participante em questão, como discente da instituição, passou a ser acompanhado por pedagogas e psicológa, visto que apresentou situação semelhante anteriormente.

Apenas três participantes do desafio enviaram relatos em formato de áudio. Os relatos recebidos são transcritos a seguir e foram de participantes cujo impacto foi, notadamente, positivo.

\section{Relato 1}

"Eu queria relatar que foi um desafio muito bom pra mim, muito produtivo, porque... no início eu não estava entendendo muito bem qual era o propósito, meio confuso as ideias, mas, assim... desde o primeiro dia fiz certinho, fiz o planejamento que me ajudou bastante. Nunca tinha feito um planejamento tão detalhado que focasse também no meu momento de lazer, no meu tempo livre... Então isso me ajudou. Eu consegui fazer coisas que a tempos eu tava planejando. Atingi todas as minhas metas, meus objetivos, consegui fazer coisas que eu gosto além das coisas que eu precisava fazer. Além disso, os podcasts "Seja otimista" e "Seja grato" foram muito bons, me ajudaram bastante para umas ideias (...)" 


\section{Relato 2}

"Bom... Depois do planejamento, eu não coloquei totalmente em prática, mas em questão de horários para cumprir (...) tenho melhorado porque o dia tem ficado maior, digamos assim. E em relação às metas, estou mais focado. Então isso melhorou bastante em relação a antes do desafio."

\section{Relato 3}

"Ótimo desafio. Desafio nota 10. (...) dizer que me ajudou mais ainda em questão de gestão de tempo e produtividade. Eu já vinha tentando organizar minhas metas, meus objetivos, minhas atividades do dia, e esse (desafio) foi mais uma ajuda, principalmente, na questão de ser otimista e de ser grato por poder fazer várias tarefas ao longo do dia. Ser otimista e buscar cada vez mais. (...) muito obrigado pela ajuda."

Por meio dos relatos, torna-se perceptível a questão das deficiências no que se refere ao planejamento que, geralemnte, exclui momentos de lazer.

Os relatos indicam que a abordagem contribuiu para a realização de melhores planejamentos, maior organização e foco nas atividades, além de favorecer o bem-estar dos participantes com exercícios relacionados ao otimismo e gratidão.

\section{CONSIDERAÇÕES FINAIS}

A proposta de abordagem do Scrum para a gestão de tempo e produtividade, integrando elementos da psicologia positiva, pode contribuir para maior produtividade e bem-estar subjetivo dos praticantes.

Os impactos identificados constituem em resultado preliminar da abordagem, sendo necessário estudo mais aprofundado. Nesse sentido, aponta-se algumas iniciativas em planejamento, como o uso do desafio (abordagem) pela psicologia da instituição de ensino e um estudo longitudinal dos impactos da abordagem do scrum associada à psicologia positiva para gestão de tempo.

O uso da abordagem pela psicologia da instituição tem por propósito instruir os discentes na gestão de tempo, organização de estudo e cuidado em relação à saúde mental.

Aplicação com os diversos discentes da instituição para a pesquisa longitudinal visa avaliar os impactos e a durabilidade do efeito ao longo do tempo.

Por fim, o fato de participantes relatarem maior controle sobre o tempo, como se o dia fosse maior, é algo que chamou atenção, visto que a sensação de controle sobre os horários proporciona certo emponderamento diante de circunstâncias que ocasionam em ansiedade. Conclui-se que a abordagem tem efeitos positivos na produtividade e saúde mental dos praticantes.

\section{REFERÊNCIAS}

Wanderson Carneiro Moreira, Anderson Reis de Sousa, and Maria do Perpétuo Socorro de Sousa Nóbrega. Mental Illness in the general population and health professionals during Covid-19: A scoping review. Texto \& Contexto - Enfermagem, 29, 00 2020. ISSN 0104-0707. doi: http://dx.doi.org/10.1590/1980-265xtce-2020-0215. URL http://www.scielo.br/scielo.php?script=sci_arttext\&pid=S010407072020000100208\&nrm=iso.

Berta Rodrigues Maia and Paulo César Dias. Ansiedade, depressão e estresse em estudantes universitários: o impacto da COVID-19. Estudos de Psicologia (Campinas), 37,
00 2020. ISSN 0103-166X. doi: 10.1590/1982-0275202037e200067. URL http://www. scielo.br/scielo.php?script=sci_arttext\&pid=S0103-166X2020000100504\&nrm=iso.

Laura Mattos. Pesquisa aponta aumento de ansiedade e tristeza em jovens na pandemia, August 2020. URL https://www1.folha.uol.com.br/cotidiano/2020/08/pesquisaaponta-aumento-de-ansiedade-e-tristeza-em-jovens-na-pandemia.shtml.

M. E. P. Seligman and M. Csikszentmihalyi. Positive psychology: An introduction. American Psychologist, 55:5 - 14, 2000. doi: 10.1037/0003-066X.55.1.5. URL https: //doi.org/10.1037/0003-066X.55.1.5

Letícia George Camalionte and Marina Pereira Rojas Boccalandro. Felicidade e bemestar na visão da psicologia positiva. Boletim - Academia Paulista de Psicologia, 37: 206 - 227, 07 2017. ISSN 1415-711X. URL http://pepsic.bvsalud.org/scielo.php? script=sci_arttext\&pid=S1415-711X2017000200004\&nrm=iso.

Cristian Zanon, Letícia Lovato Dellazzana-Zanon, Solange Muglia Wechsler, Rodrigo Rodrigues Fabretti, and Karina Nalevaiko da Rocha. COVID-19: implicações e aplicações da Psicologia Positiva em tempos de pandemia. Estudos de Psicologia (Campinas), 37:1-13, 00 2020. ISSN 0103-166X. URL http://www.scielo.br/scielo. php?script=sci_arttext\&pid=S0103-166X2020000100506\&nrm=iso.

Kennon M. Sheldon and Sonja Lyubomirsky. Revisiting the sustainable happiness model and pie chart: Can happiness be successfully pursued? The fournal of Positive Psychology, 0(0):1-10, 2019. doi: 10.1080/17439760.2019.1689421.

SCRUMStudy. A Guide to the SCRUM BODY OF KNOWLEDGE (SBOKTMGUIDE) 2016 Edition. VMEduc, Phoenix, AZ, 1nd. edition, 2016. ISBN 978-0-9899252-0-4.

Ken Schwaber and Jeff Sutherlandn. Guia do Scrum. Um guia definitivo para o Scrum: As regras do jogo. Scrum.org and Scrum Inc., 1nd. edition, 2017.

Cesar Brod. Scrum - Guia Prático para Projetos Ágeis. Novatec, São Paulo, SP, 2nd. edition, 2015. ISBN 978-85-7522-441-0.

David Starr and Eleanor Starr. Agile practices for families: Iterating with children and parents, 2008.

B. Feiler. The Secrets of Happy Families: Improve Your Mornings, Rethink Family Dinner, Fight Smarter, Go Out and Play and Much More. Little, Brown Book Group, 2013. ISBN 9780349402239. URL https://books.google.com.br/books?id=lGneszdK2rYC.

Kent Beck, Mike Beedle, Arie van Bennekum, Alistair Cockburn, Ward Cunningham, Martin Fowler, James Grenning, Jim Highsmith, Andrew Hunt, Ron Jeffries, Jon Kern, Brian Marick, Robert C. Martin, Steve Mellor, Ken Schwaber, Jeff Sutherland, and Dave ThomasIngo Lütkebohle. Manifesto para desenvolvimento Ágil de software. https://agilemanifesto.org/iso/ptbr/manifesto.html, 2001. [Online; accessed 20-November-2020].

J. Sutherland. Scrum: a arte de fazer o dobro do trabalho na metade do tempo, pages 117-123. Leya Brasil, 2016. ISBN 9788544104712. URL https://books.google.com. br/books?id=Q1G2DQAAQBAJ.

Caio Souza. Acompanhando projetos pessoais com o scrum, oct 2018. URL https: //blog.myscrumhalf.com/acompanhando-projetos-pessoais-com-o-scrum/.

Arno Delhij, Rini van Solingen, and Willy Wijnands. O Guia eduScrum. As regras do jogo. 1nd. edition, 2016.

D. Allen. A arte de fazer acontecer: uma fórmula anti-stress para estabelecer prioridades e entregar soluções no prazo. ELSEVIER EDITORA, 2005. ISBN 9788535219081. URL https://books.google.com.br/books?id=TW 8IL7QOaYC.

Shawn Achor. O feito Harvard de Ser Feliz. Saraiva Educação S.A., 2017. ISBN 9788502180284. URL https://books.google.com.br/books?id=edJiDwAAQBAJ. 